\title{
Estudio de efectos de sitio en la Región de Coquimbo durante el terremoto de Illapel $\mathrm{M}_{\mathrm{w}} 8.3$ de 2015
}

\section{Study of site effects in the Coquimbo Region due to the 2015 Mw 8.3 Illapel, Chile, Earthquake}

Fecha de entrega: 6 de diciembre 2016 Fecha de aceptación: 24 de abril 2017

\section{José Fernández ${ }^{1}$, César Pastén ${ }^{1}$, Sergio Ruiz² y Felipe Leyton ${ }^{3}$}

\author{
${ }^{1}$ Departamento de Ingeniería Civil, Universidad de Chile, Av. Blanco Encalada 2002, Santiago, Chile, jose.fernandez@ing.uchile.cl, \\ cpasten@ing.uchile.cl \\ ${ }^{2}$ Departamento de Geofísica, Universidad de Chile, Av. Blanco Encalada 2002, Santiago, Chile, sruiz@dgf.uchile.cl \\ ${ }^{3}$ Centro Sismológico Nacional, Universidad de Chile, Av. Beaucheff 1225, Santiago, Chile, leyton@csn.uchile.cl
}

Este trabajo tiene como objetivo determinar efectos de sitio en las localidades de la Región de Coquimbo producidos por el terremoto Illapel $M_{w} 8.3$ de 2015, particularmente aquellas que cuentan con estaciones sismológicas. Se relaciona la respuesta sísmica y la distribución de daños durante el evento con la geomorfología y geología de los sitios. Para ello se emplearon razones espectrales $H / V$ (HVSR) calculadas a partir de registros de aceleraciones de sismos con magnitud moderada a alta $\left(M_{w} \geq 5\right)$, ocurridos en una ventana temporal de tres años en torno al evento principal. Por otra parte, el método HVSR fue también aplicado a mediciones pasivas de vibraciones ambientales de corta duración (20 a 30 minutos), realizadas con sismógrafos de $4.5 \mathrm{~Hz}$. A partir de ambas variantes del método, se obtuvieron razones espectrales en función del periodo, caracterizando dinámicamente los suelos por medio de su periodo predominante de vibración. Esta información se contrastó con la geología y geomorfología de las localidades, para explicar los efectos de sitio en cada una de ellas. Finalmente, se compararon los resultados con estimaciones de intensidad calculadas a partir de un catastro de viviendas dañadas producto del terremoto, elaborado por instituciones gubernamentales. Se concluye que las estaciones sismológicas de la Región de Coquimbo se encuentran en su mayoría sobre depósitos de suelo rígido o en afloramientos rocosos, lo que es consistente con que las intensidades MSK alcanzaran valores máximos de 6.5 en la Región.

Palabras clave: efectos de sitio, razones espectrales $H / V$, intensidades MSK
This work aims to determine site effects in the Coquimbo Region due to the $2015 M_{w} 8.3$ Illapel earthquake, particularly in towns with seismological stations. In addition, it seeks to relate the seismic response and the distribution of damage during the event with the geomorphology and geology of the sites. This study used H/V spectral ratios (HVSR) applied to acceleration records of moderate to high magnitude earthquakes $\left(M_{w} \geq 5\right)$ occurred in a time window of three years around the main event. Moreover, the HVSR method was also applied to seismic ambient vibration records (20 to 30 minutes long), recorded by $4.5 \mathrm{~Hz}$ seismographs. Both variations of the method generated spectral ratios as a function of the period, allowing the dynamic characterization of the soil deposits through their predominant vibration period. This information is complemented with the geology and geomorphology of the studied zones in order to explain the site effects in each one. Finally, the results are compared with calculated MSK intensities from a survey of housing damage caused by the earthquake, implemented by government institutions. Our analyses conclude that the seismological stations of the Coquimbo Region are mostly over rigid soil deposits and rock outcrops, which is consistent with the MSK intensities that reached maximum values of 6.5 in the Region.

Keywords: site effects, HV spectral ratios, MSK intensities

\section{Introducción}

Chile ha sido azotado históricamente por terremotos de gran magnitud $\left(M_{\mathrm{w}}>8\right)$. En particular, desde 2010 se han sumado los terremotos del Maule $2010 M_{\mathrm{w}} 8.8$, Iquique $2014 M_{\mathrm{w}} 8.2$ e Illapel $2015 M_{\mathrm{w}} 8.3$. El terremoto de Illapel $M_{\text {w }} 8.3$ ocurrió el 16 de septiembre de 2015 a las 19:54:31 
hora local, con un hipocentro ubicado en $31.64^{\circ} \mathrm{S}$ y $71.75^{\circ} \mathrm{W}$ y $23 \mathrm{~km}$ de profundidad (Centro Sismológico Nacional, $\mathrm{CSN})$. Este terremoto ocurre en la parte superficial del contacto de placas con una ruptura Sur-Norte (Ruiz et al., 2016). La intensidad reportada por la Oficina Nacional de Emergencia del Ministerio del Interior y Seguridad Pública (ONEMI) en escala Mercalli Modificada (MM) fue de VIII en la Región de Coquimbo y V en la Región de Atacama, lo que implica una rápida atenuación del terremoto hacia el norte. Además, tanto en las Regiones de Valparaíso como Metropolitana, la intensidad fue de VII en la misma escala. El evento afectó a 7285 viviendas (Gobierno de Chile, 2015) y en conjunto con el tsunami posterior dejaron 13 víctimas fatales (GEER, 2015). Algunos de los registros de aceleraciones asociados al terremoto se exhiben en la Figura 1 , mientras que las aceleraciones máximas PGA registradas se muestran en la Tabla 1.

En la zona del terremoto de Illapel 2015 han ocurrido previamente los terremotos de Illapel 1943 y Punitaqui 1997 (Beck et al., 1998; Lemoine et al., 2001; Pardo et al., 2002). El primero ocurrió el día 6 de abril de 1943 a las 12:07 hora local, con epicentro de coordenadas $30.75^{\circ} \mathrm{S}$ y $72.00^{\circ} \mathrm{W}$, frente a la desembocadura del Río Limarí y tuvo una magnitud $M_{\mathrm{w}}=7.8$ (Beck et al., 1998). Su profundidad fue de $55 \mathrm{~km}$ y el tamaño de la ruptura alcanzó los $200 \mathrm{~km}$ aproximadamente (Beck et al., 1998), siendo similar en ubicación, magnitud e incluso en longitud de ruptura al terremoto de Illapel 2015. En cuanto a sus efectos, se cuentan 12 víctimas fatales, 49 heridos y más de 23000 damnificados (E1 Mercurio, 2007), además de daños severos a la infraestructura. Localidades como Combarbalá, Punitaqui, Limarí y otras quedaron con el $80 \%$ de sus viviendas inhabitables (Piñones, 2002). Con la información de la zona, se confeccionó un mapa de isosistas (Greve, 1946) que reveló intensidades V (medidas en una escala de intensidades con valor máximo VI) en torno al epicentro, es decir, Ovalle, Combarbalá e Illapel, mientras que Ovalle registró una intensidad de VIII en escala MSK. Se debe considerar que, durante la época, la población se concentraba en los valles centrales de la depresión intermedia de la Región y no en la costa (Piñones, 2002).

El 14 de octubre de 1997 a las 22:03:30 hora local se produjo el terremoto de Punitaqui, de tipo intraplaca de profundidad intermedia en la Placa de Nazca. El epicentro se ubicó a $10 \mathrm{~km}$ al norte de Punitaqui $\left(31.02^{\circ} \mathrm{S}\right.$ y $\left.71.23^{\circ} \mathrm{W}\right)$ y registró una magnitud $M_{\mathrm{w}}=7.1$ (Lemoine et al., 2001; Pardo et al., 2002). La estación más cercana estaba en Illapel y registró una aceleración máxima de $0.35 \mathrm{~g}$ en la componente horizontal $\mathrm{N} 70^{\circ} \mathrm{E}$ (Díaz, 2001). De acuerdo con reportes de ONEMI y Carabineros, el sismo fue sensible desde Taltal (Región de Antofagasta) hasta Angol (Región de la Araucanía) con intensidad II en escala MM en ambas localidades (ONEMI, 1997). A partir de los daños evidenciados en el adobe, se calcularon intensidades en escala MSK que variaron entre V y VIII en las distintas localidades de la Región de Coquimbo (Díaz, 2001).

Considerando la respuesta sísmica de la región, el objetivo principal de este trabajo es relacionar el nivel de daños percibidos y registrados producto del terremoto $M_{\mathrm{w}} 8.4$ de 2015 con la rigidez de los depósitos de suelo. Para ello, se caracterizaron los suelos de las distintas comunas, determinando su geología y calculando razones espectrales H/V a partir de registros sísmicos (Lermo y Chávez-García, 1993), obtenidos en las estaciones sismológicas mostradas en la Figura 1, y a partir de registros de vibraciones ambientales (Nakamura, 1989). Paralelamente, se realizó un estudio de daños enfocado a obtener intensidades en la escala MSK (Medvedev et al., 1964) mediante la metodología propuesta por Monge y Astroza (1989), la cual contrasta la distribución de los distintos grados de daños en las estructuras con los grados de intensidad de esta escala.

Tabla 1: Aceleraciones máximas PGA del terremoto de Illapel 2015 registradas en las estaciones de la Región de Coquimbo

\begin{tabular}{|c|c|c|c|c|}
\hline \multicolumn{2}{|c|}{ Estación } & \multicolumn{3}{c|}{ PGA, g } \\
\hline Código & Ubicación & NS & EW & Z \\
\hline C01O & La Serena & 0.18 & 0.15 & 0.12 \\
\hline C09O & La Higuera & 0.19 & 0.19 & 0.09 \\
\hline C11O & Monte Patria & 0.71 & 0.83 & 0.48 \\
\hline C19O & Las Tacas & 0.11 & 0.12 & 0.10 \\
\hline C20O & Hospital Coquimbo & 0.25 & 0.26 & 0.18 \\
\hline
\end{tabular}

\begin{tabular}{|c|c|c|c|c|}
\hline \multicolumn{2}{|c|}{ Estación } & \multicolumn{3}{c|}{ PGA, g } \\
\hline Código & Ubicación & NS & EW & Z \\
\hline C22O & UCN Guayacán & 0.13 & 0.09 & 0.07 \\
\hline C26O & Tongoy & 0.23 & 0.36 & 0.13 \\
\hline C33O & Estadio La Portada & 0.11 & 0.14 & 0.07 \\
\hline CO03 & Pedregal & 0.29 & 0.35 & 0.20 \\
\hline GO04 & Obs. Cerro Tololo & 0.34 & 0.24 & 0.16 \\
\hline
\end{tabular}



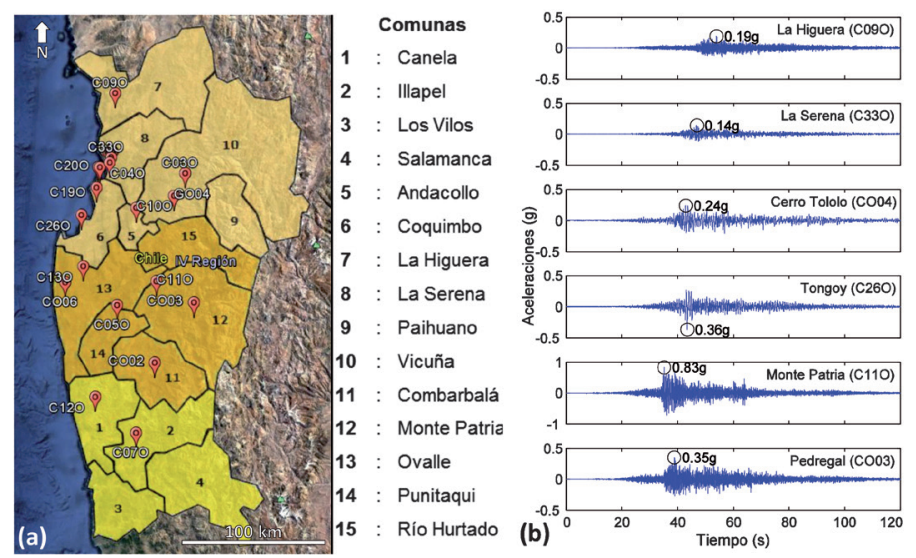

Figura 1: Región de Coquimbo. a) Distribución de estaciones sismológicas en las comunas de la Región y b) registros de aceleraciones del terremoto de Illapel 2015 en la componente EW de 6 estaciones ubicadas de norte a sur.

Para esto, se utilizó un catastro de daños elaborado por el Ministerio de Vivienda y Urbanismo MINVU después del terremoto (comunicación personal, solicitud mediante Ley de Transparencia $\mathrm{N}^{\circ} 20.285,29$ de julio de 2016), donde se califica la situación de las viviendas de la región y se compara con el total de viviendas reportadas por el Censo no oficial de 2012.

\section{Caracterización de suelos}

La caracterización de los suelos se realizó por medio de la geología y el método de las razones espectrales H/V (HVSR) aplicado tanto a registros sísmicos como a registros de vibraciones ambientales.

\section{Geología}

De acuerdo con la descripción geomorfológica realizada por Börgel (1983), la Región de Coquimbo está caracterizada fundamentalmente por la presencia de dos grandes unidades morfológicas. La primera se manifiesta en la fuerte presencia de cordones montañosos transversales que conectan la Cordillera de Los Andes con la Cordillera de la Costa, promoviendo la formación intensa de valles en la Depresión Intermedia. Estos cordones adquieren alturas de 600 a 1000 msnm, en disposición E-W principalmente, y se ven originados por la erosión de quebradas y ríos, destacando las cuencas de Elqui, Limarí y Choapa. Así, la región se caracteriza por presentar una compleja faja interna de relieve montañoso de $50 \mathrm{~km}$ de ancho aproximadamente, además de pequeñas cuencas tectónicas y corredores longitudinales N-S. Por otra parte, las planicies litorales pueden ser de 3 tipos: fluviales, marinas o fluviomarinas. Esta morfología alcanza un desarrollo de $30 \mathrm{~km}$ de ancho entre La Serena y Tongoy, gracias a la desembocadura de ríos, con alto nivel de depositación de materiales, formando dunas y playas continentales. Dentro del contexto geomorfológico anterior y a partir de cartas geológicas (Aguirre y Egert, 1970; Moscoso et al., 1982; Mpodozis y Cornejo, 1988; Rivano y Sepúlveda, 1991; Thomas, 1967) y otros trabajos (Rosales, 1988; García, 1999), es posible obtener la geología local para cada estación sismológica. Esta información se muestra en la Tabla 2, de donde es posible distinguir que gran parte de las localidades se encuentra sobre depósitos aluviales o similares de época cuaternaria, lo que coincide con el asentamiento poblacional de la región en torno a los valles fértiles de los ríos de la zona. Por otro lado, las zonas costeras están principalmente sobre rocas antiguas (jurásicas) lo que es concordante con la edad de la Cordillera de la Costa, pero también sobre terrazas de deposición marina y fluvial.

\section{Razones espectrales $\mathrm{H} / \mathrm{V}$}

Las curvas HVSR se calcularon a partir de registros de aceleraciones medidos en las estaciones de la Tabla 2 y mediciones de vibraciones ambientales. Los registros sísmicos se obtuvieron entre enero de 2013 y marzo de 2016 y corresponden a eventos de magnitud igual o superior a 5. Los registros de vibraciones ambientales se obtuvieron con sismógrafos Tromino ${ }^{\circledR}$ de 3 componentes y $4.5 \mathrm{~Hz}$ de frecuencia natural en campañas realizadas por el Centro Sismológico Nacional y los Departamentos de Ingeniería Civil y Geofísica (Universidad de Chile). Las razones espectrales H/V (Lermo y Chávez-García, 1993) se obtuvieron con el software Geopsy ${ }^{\circledR}$ para frecuencias entre 0.2 y $25 \mathrm{~Hz}$ seleccionando ventanas de $30 \mathrm{~s}$ de largo en la señal y utilizando filtro propuesto por Konno y Ohmachi (1998) con una constante de suavizado $b=40$.

El procesamiento de registros sísmicos se realizó aplicando filtros tipo Butterworth de orden 4 entre 0.2 y $25 \mathrm{~Hz}$ a cada una de las componentes horizontales y la vertical. Luego, se empleó la transformada de Fourier suavizada con una media móvil de vecindad $0.25 \mathrm{~Hz}$ para cada componente, se combinaron las horizontales con una media geométrica y se dividió la componente horizontal combinada por la componente vertical para obtener las razones espectrales 
Tabla 2: Geología de las localidades con estaciones sismológicas

\begin{tabular}{|c|c|c|c|c|}
\hline Comuna & Localidad & $\begin{array}{c}\text { Estación } \\
\text { Sismológica }\end{array}$ & Geología & Descripción \\
\hline \multirow{4}{*}{ Coquimbo } & Las Tacas & $\mathrm{C} 19 \mathrm{O}$ & $\mathrm{Kt}(\mathrm{Kg} / \mathrm{QTm})$ & $\begin{array}{l}\text { Rocas volcánicas andesíticas con brechas y tobas } \\
\text { (riolíticas) }\end{array}$ \\
\hline & Coquimbo & $\mathrm{C} 20 \mathrm{O}$ & Tec (JKg/Qal) & $\begin{array}{l}\text { Sedimentos marinos aterrazados, areniscas, } \\
\text { calcoarenitas }\end{array}$ \\
\hline & Coquimbo & $\mathrm{C} 22 \mathrm{O}$ & $\mathrm{JKg}$ & Granito \\
\hline & Tongoy & $\mathrm{C} 26 \mathrm{O}$ & Ja (Qal) & $\begin{array}{l}\text { Rocas volcánicas andesíticas y sedimentarias } \\
\text { clásticas continentales }\end{array}$ \\
\hline La Higuera & La Higuera & $\mathrm{C} 09 \mathrm{O}$ & Tega/Qal (Kg) & $\begin{array}{l}\text { Gravas de Atacama, poco consolidadas, incluyendo } \\
\text { depósitos de ríos aterrazados }\end{array}$ \\
\hline Vicuña & Cerro Tololo & GO04 & Kle (Kv) & $\begin{array}{l}\text { Rocas volcánicas andesíticas basálticas y riolíticas, } \\
\text { intercalada con sedimentarias }\end{array}$ \\
\hline \multirow{2}{*}{ La Serena } & Las Compañías & $\mathrm{C} 01 \mathrm{O}$ & Qal & Depósitos aluviales cuaternarios \\
\hline & La Serena & $\mathrm{C} 33 \mathrm{O}$ & Qal & Depósitos aluviales cuaternarios \\
\hline Combarbalá & Combarbalá & $\mathrm{CO} 02$ & Qal (Kqmc/Kqmt) & $\begin{array}{l}\text { Depósitos aluviales cuaternarios y conglomerados, } \\
\text { tobas y brechas }\end{array}$ \\
\hline \multirow[b]{2}{*}{ Monte Patria } & Monte Patria & $\mathrm{C} 11 \mathrm{O}$ & Qal (Kv) & Depósitos aluviales cuaternarios y rocas volcánicas \\
\hline & Pedregal & $\mathrm{CO} 03$ & Qac (Kv/ah) & $\begin{array}{l}\text { Sedimentos aluviales y coluviales, depósitos de } \\
\text { relleno de valles }\end{array}$ \\
\hline \multirow{3}{*}{ Ovalle } & Ovalle & - & Qal/Qc (Kra/Ka) & $\begin{array}{l}\text { Depósitos aluviales cuaternarios y sedimentos } \\
\text { continentales }\end{array}$ \\
\hline & Limarí & $\mathrm{C} 13 \mathrm{O}$ & Qal (Qc) & $\begin{array}{l}\text { Depósitos aluviales cuaternarios y sedimentos } \\
\text { continentales }\end{array}$ \\
\hline & Angostura & $\mathrm{CO} 06$ & $\operatorname{Jgd}(\mathrm{Jdn})$ & Granito a granodiorita, diorita gnéisica \\
\hline Punitaqui & Punitaqui & $\mathrm{C} 05 \mathrm{O}$ & Qc (Kg) & Sedimentos continentales y granito \\
\hline Canela & Canela Baja & $\mathrm{C} 12 \mathrm{O}$ & Qc/Jmi4 & $\begin{array}{l}\begin{array}{l}\text { Sedimentos continentales } \\
\text { (granodioritas y tonalitas) }\end{array} \\
\end{array}$ \\
\hline Illapel & Illapel & $\mathrm{C} 07 \mathrm{O}$ & Qac/Tc (Ki1) & $\begin{array}{l}\text { Sedimentos aluviales y coluviales con conglomerados, } \\
\text { areniscas, brechas, limolitas }\end{array}$ \\
\hline Los Vilos & Los Vilos & - & Qal (Jmil/Dca) & $\begin{array}{l}\text { Depósitos aluviales además de lutitas, areniscas } \\
\text { y estratificación gradada. Monzogranitos y } \\
\text { sienogranitos. }\end{array}$ \\
\hline Salamanca & Salamanca & - & Qac (Ki1) & $\begin{array}{l}\text { Sedimentos aluviales y coluviales junto con } \\
\text { granodioritas (intrusivos) }\end{array}$ \\
\hline
\end{tabular}

HVSR. En la Figura 2 se muestran las HVSR en función del periodo y la cantidad de eventos considerados en cada estación.

De acuerdo con la propuesta de clasificación de suelos de Idini (2016), se consideran suelos rígidos aquellos que presentan HVSR planas para todo el rango de periodos o que poseen un periodo predominante de vibración menor a 0.4 s. Según estos criterios, es posible observar que en la Región de Coquimbo los suelos de las estaciones ubicadas en Las Tacas C19O, campus Guayacán de la Universidad Católica del Norte C22O, La Higuera C09O, estadio La Portada en La Serena C33O, Combarbalá CO02, Monte Patria C11O, sector El Pedregal CO03, Angostura CO06, Punitaqui CO05, Canela Baja C12O e Illapel C07O son rígidos, a diferencia de los suelos de las estaciones Hospital de Coquimbo C20O, Tongoy C26O, Observatorio Cerro Tololo GO04, Las Compañías en La Serena C01O y el sector de Limarí C13O, que no cumplen con estas condiciones. 

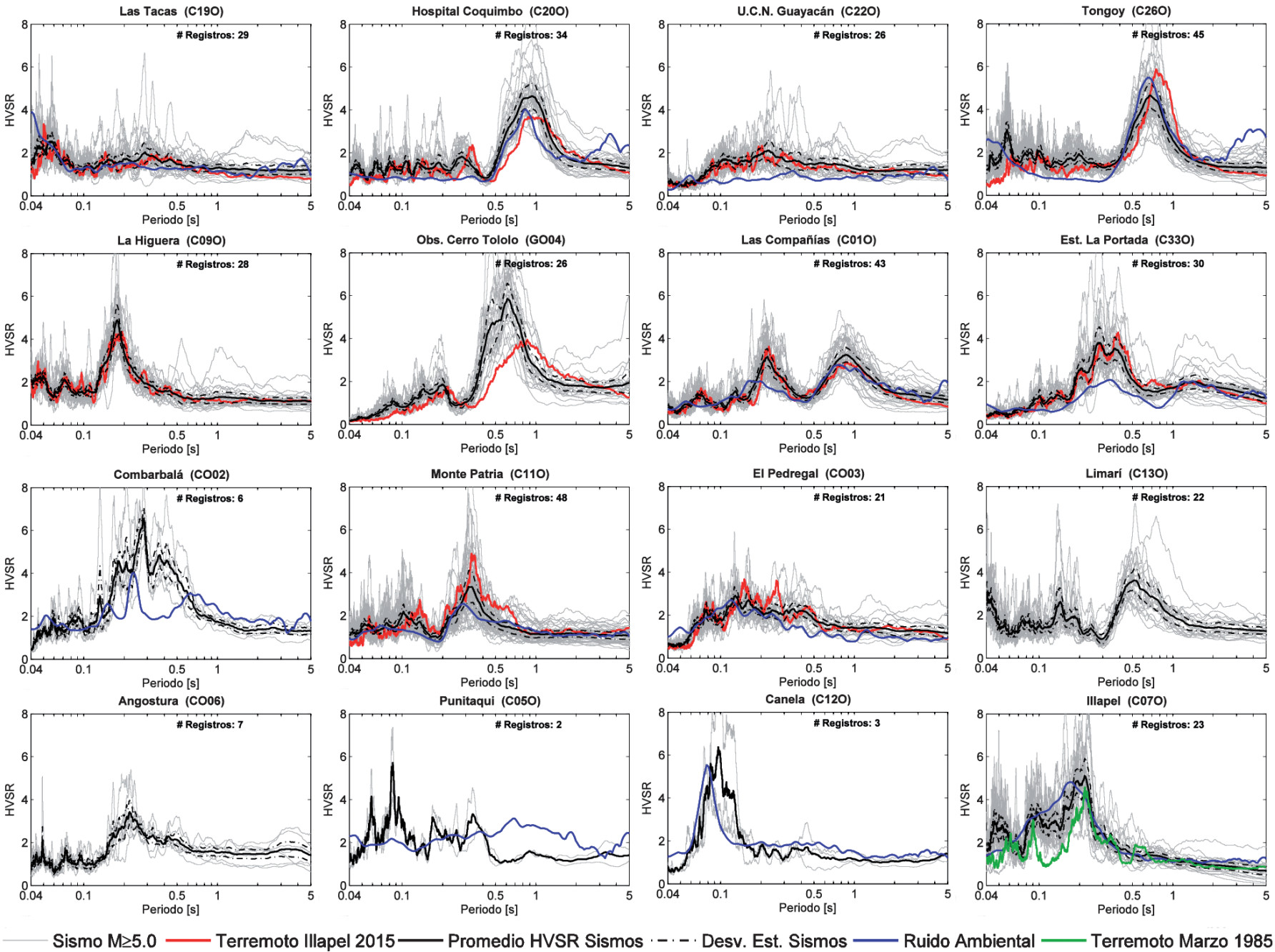

Figura 2: Resultados de razones espectrales H/V para las 16 estaciones sismológicas en la Región de Coquimbo con actividad registrada entre 2013 y 2016. Diez de estas estaciones registraron el Terremoto de Illapel 2015 y 12 cuentan con mediciones de vibraciones ambientales

En la Figura 2 se puede observar consistencia en la estimación de los periodos predominantes de vibración con ambas metodologías de razones espectrales, con la sola excepción de Punitaqui $\mathrm{C} 05 \mathrm{O}$, que sólo cuenta con dos registros sísmicos. A modo de comparación, en la Figura 2 se muestra también la razón espectral del registro del terremoto de Valparaíso 1985 en la estación de Illapel, perteneciente a la Red de Cobertura Nacional de Acelerógrafos de la Universidad de Chile (RENADIC), que coincide con los resultados de sismos de menor magnitud registrados en la estación $\mathrm{C} 07 \mathrm{O}$.

Adicionalmente, se estimaron algunos perfiles de velocidad de onda de corte mediante correlaciones cruzadas de ruido sísmico (Sáez, 2016) e inversiones con el software Geopsy®, utilizando generación Monte Carlo de perfiles y considerando el ajuste tanto de curvas de dispersión como de HVSR. Estos resultados dan cuenta de depósitos de suelos rígidos con un rápido aumento de $V_{\mathrm{s}}$ en profundidad. La Figura 3 muestra los resultados para las estaciones de Limarí C13O y Tongoy C26O, donde se muestran los perfiles que poseen como máximo 1.5 veces el mínimo error de ajuste (misfit) con respecto a las curvas de dispersión y las HVSR medidas, lo que representa una confiabilidad estadística del orden del 90\%.

\section{Registros de daños}

El trabajo realizado en torno a los daños dice relación con el cálculo de intensidades en la escala Medvedev - Sponheuer - Karnik MSK (Medvedev et al., 1964), de acuerdo a las adaptaciones al caso de Chile realizadas 

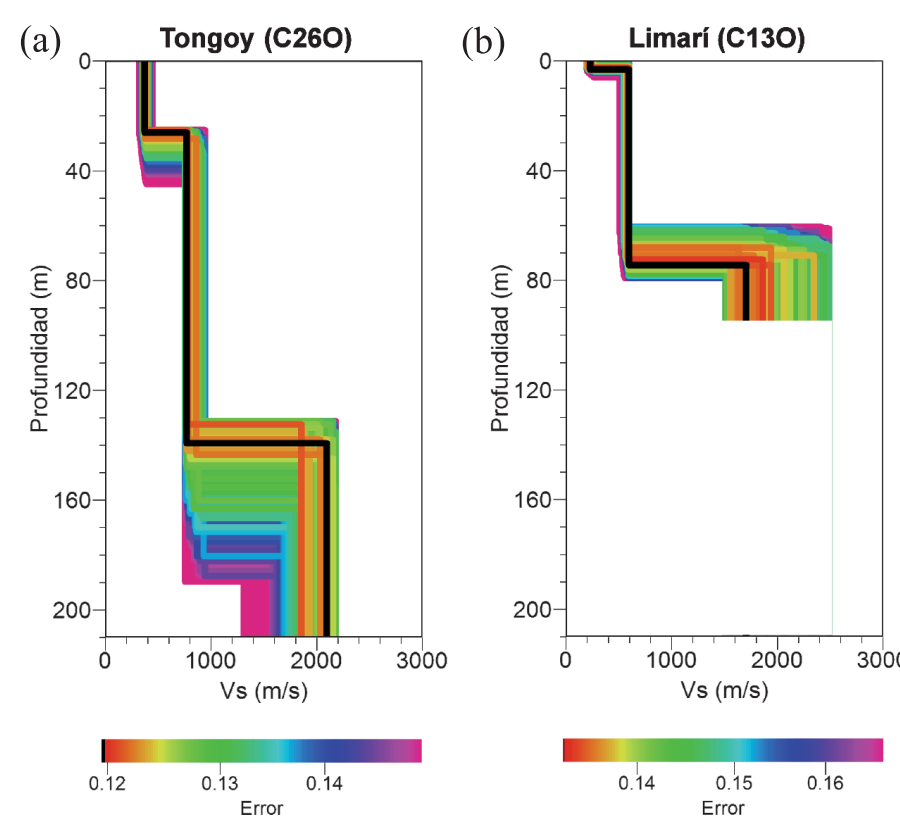

Figura 3: Perfiles de velocidad de onda de corte $\mathrm{V}_{\mathrm{s}}$ en las estaciones de: a) Limarí C13O y b) Tongoy C26O. La escala de colores representa el error de ajuste asociado a cada perfil y la curva negra el perfil con menor error. La profundidad máxima de los perfiles está dada por la máxima longitud de onda que captura el método de las correlaciones cruzadas (Sáez, 2016). por Monge y Astroza (1989). La metodología consiste en determinar el grado de intensidad a partir de la distribución de daños observados en viviendas de cierta materialidad en una localidad. El trabajo original detalla 6 grados de daños, desde la categoría N0: sin daños, hasta la N5: caída de más de un muro. Esta evaluación se realiza por medio de visitas a terreno y encuestas en una zona aleatoria y representativa de la localidad en estudio.

La información de daño disponible del terremoto de Illapel 2015 corresponde a un catastro realizado por el MINVU, con el objetivo de distribuir recursos para la reparación y reconstrucción de viviendas. Este catastro contempla 5 categorías de daño: $\sin$ daños, daños reparables leves, daños reparables moderados, daños reparables mayores y daños no reparables, e incluye distintos tipos de materialidad de las viviendas. Por esta razón, se adaptó el trabajo realizado por Díaz (2001), quien homologó ambas escalas de daños para lograr consistencia en la asignación de intensidades MSK al adobe en la Región de Coquimbo después del terremoto de Punitaqui 1997. La Figura 4 muestra fotografías de estructuras dañadas en la Región de Coquimbo.

Tabla 3: Localidades, daños e intensidad asociadas a los terremotos de Punitaqui 1997 (Díaz, 2001) e Illapel 2015

\begin{tabular}{|c|c|c|c|c|c|c|c|c|c|c|}
\hline \multirow[t]{2}{*}{ Comuna } & \multirow[t]{2}{*}{ Localidad } & \multirow[t]{2}{*}{ Estación } & \multirow{2}{*}{$\begin{array}{c}\text { MSK } \\
1997\end{array}$} & \multirow{2}{*}{$\begin{array}{l}\text { Censo } \\
2012\end{array}$} & \multicolumn{5}{|c|}{ Daños en adobe 2015} & \multirow{2}{*}{$\begin{array}{l}\text { MSK } \\
2015 \\
\end{array}$} \\
\hline & & & & & D0 & D1 & D2 & D3 & D4 & \\
\hline \multirow{4}{*}{ Coquimbo } & Las Tacas & C19O & $\mathrm{S} / \mathrm{I}$ & \multirow{4}{*}{2633} & \multirow{4}{*}{2577} & \multirow{4}{*}{10} & \multirow{4}{*}{12} & \multirow{4}{*}{22} & \multirow{4}{*}{12} & $<5$ \\
\hline & Coquimbo & $\mathrm{C} 20 \mathrm{O}$ & 5.0 & & & & & & & \\
\hline & Coquimbo & $\mathrm{C} 22 \mathrm{O}$ & 5.0 & & & & & & & \\
\hline & Tongoy & $\mathrm{C} 26 \mathrm{O}$ & 5.5 & & & & & & & \\
\hline La Higuera & La Higuera & $\mathrm{C} 09 \mathrm{O}$ & 5.5 & 79 & - & - & - & - & - & $\mathrm{S} / \mathrm{I}$ \\
\hline Vicuña & Cerro Tololo & GO04 & N/A & 1044 & 848 & 17 & 57 & 79 & 43 & $<5$ \\
\hline \multirow{2}{*}{ La Serena } & Las Compañías & $\mathrm{C} 01 \mathrm{O}$ & 5.0 & \multirow{2}{*}{1801} & \multirow{2}{*}{1752} & \multirow{2}{*}{4} & \multirow{2}{*}{6} & \multirow{2}{*}{16} & \multirow{2}{*}{23} & $<5$ \\
\hline & La Serena & $\mathrm{C} 33 \mathrm{O}$ & 5.0 & & & & & & & \\
\hline Combarbalá & Combarbalá & $\mathrm{CO} 02$ & 6.5 & 1292 & 946 & 25 & 55 & 84 & 182 & 5.5 \\
\hline \multirow{2}{*}{ Monte Patria } & Monte Patria & $\mathrm{C} 11 \mathrm{O}$ & 6.5 & \multirow{2}{*}{1618} & \multirow{2}{*}{1123} & \multirow{2}{*}{35} & \multirow{2}{*}{67} & \multirow{2}{*}{131} & \multirow{2}{*}{262} & 6.0 \\
\hline & Pedregal & $\mathrm{CO} 03$ & 6.5 & & & & & & & \\
\hline \multirow{3}{*}{ Ovalle } & Ovalle & - & 7.0 & \multirow{3}{*}{1738} & \multirow{3}{*}{1468} & \multirow{3}{*}{22} & \multirow{3}{*}{51} & & & $<5$ \\
\hline & Limarí & $\mathrm{C} 13 \mathrm{O}$ & 5.0 & & & & & 90 & 107 & \\
\hline & Angostura & $\mathrm{CO} 06$ & $\mathrm{~S} / \mathrm{I}$ & & & & & & & \\
\hline Punitaqui & Punitaqui & $\mathrm{C} 05 \mathrm{O}$ & 7.5 & 473 & 369 & 6 & 17 & 39 & 42 & 5.0 \\
\hline Canela & Canela Baja & $\mathrm{C} 12 \mathrm{O}$ & 5.5 & 987 & 501 & 33 & 72 & 123 & 258 & 6.5 \\
\hline Illapel & Illapel & $\mathrm{C} 07 \mathrm{O}$ & 6.5 & 473 & 298 & 17 & 32 & 41 & 85 & 6.0 \\
\hline Los Vilos & Los Vilos & - & $\mathrm{S} / \mathrm{I}$ & 276 & 271 & 1 & 1 & 2 & 1 & $<5$ \\
\hline Salamanca & Salamanca & - & 6.5 & 378 & 224 & 4 & 24 & 48 & 78 & 6.0 \\
\hline
\end{tabular}

D0: sin daño, D1: daño leve, D2: daño moderado, D3: daño mayor, D4: daño irreparable, S/I: sin información, N/A: no aplica 

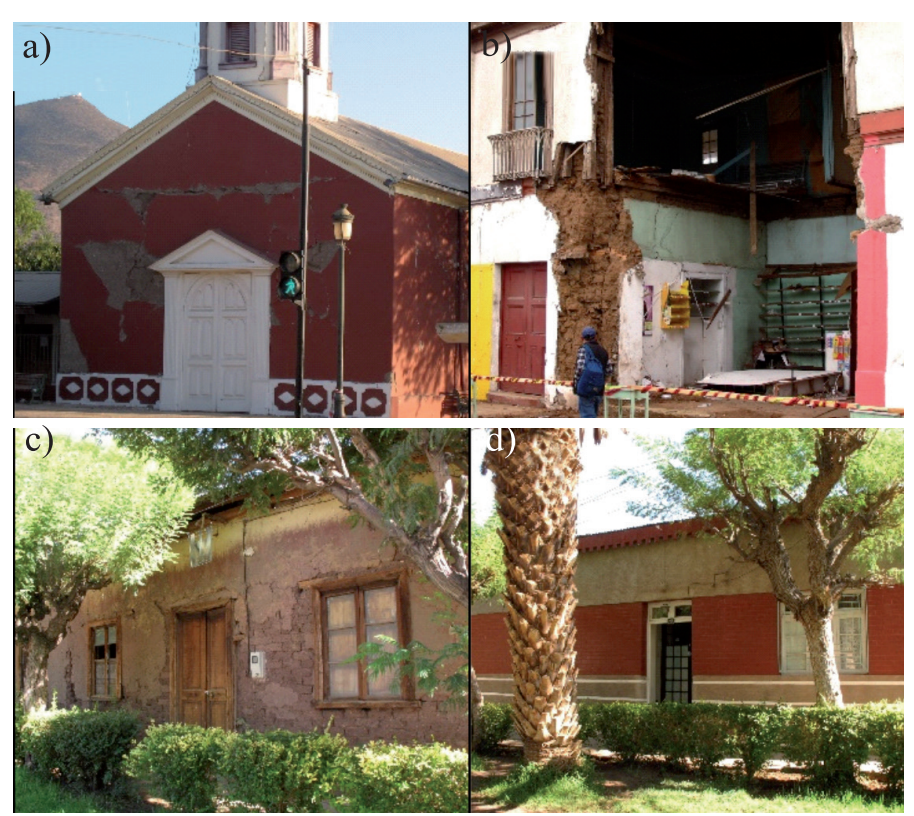

Figura 4: Fotografías de estructuras dañadas en localidades de la Región de Coquimbo tomadas en enero de 2016. a) Iglesia de Monte Patria, b) derrumbe de vivienda en Illapel, c) y d) viviendas contiguas en Combarbalá de adobe, con y sin daños, respectivamente.

El procedimiento para determinar la intensidad MSK consistió en obtener la cantidad de viviendas dañadas del catastro del MINVU, complementar con el total de viviendas obtenido del Censo 2012 y contrastar los porcentajes de distribución de daños con las curvas patrón de intensidades homologadas. Este análisis se realizó sólo para viviendas de adobe, pues presentan un mayor nivel de daño y porque la homologación solo se encuentra disponible para este caso. Además, las estructuras de adobe manifiestan un comportamiento rígido con periodos predominantes de vibración menores a $0.3 \mathrm{~s}$ (Morales et al., 1980), por lo que se espera que sean más sensibles a los períodos predominantes de los suelos reportados en la Figura 2. Las intensidades calculadas con esta metodología se muestran en la Tabla 3 y se comparan con las reportadas por Díaz (2001) para el terremoto de Punitaqui de 1997. Los resultados muestran que las intensidades percibidas por el adobe para el terremoto de Punitaqui son mayores que para el de Illapel 2015 en la mayoría de las comunas analizadas.

De acuerdo a los Censos de 1992, 2002 y 2012, las viviendas en la Región pasaron de 115986 a 255613 en 20 años, concentrándose principalmente en las comunas de La Serena y Coquimbo (23 y 24\% en 1992, 26 y 26\% en 2002 y 28 y $27 \%$ en 2012, respectivamente), seguidas de Ovalle y Monte Patria (14 y 5\% en 2012, respectivamente). Esta condición genera un desbalance en la interpretación de la materialidad representativa de la región. Considerando las dos comunas más grandes en 1992, el adobe era el material predominante (34\%), seguido de la madera (31\%). En 2012 , el escenario cambió y la albañilería representaba el $47 \%$ de las viviendas, seguida de la madera con un $24 \%$ y el adobe con un $9 \%$.

Si bien es cierto que el Censo de 2012 no es oficial por problemas metodológicos, los resultados de materialidad pueden ser confiables en cuanto el instrumento obliga el registro de las viviendas sin importar la cantidad de ocupantes. El levantamiento de información del MINVU corresponde a sectores identificados previamente con algún tipo de daño, con énfasis en zonas rurales, y complementado con peticiones de la población afectada. Esto puede generar un sesgo de la información al no tener cobertura adecuada ni aleatoriedad de la muestra.

Contrastando la totalidad de viviendas con la cantidad dañada según la encuesta MINVU, las comunas más afectadas fueron Canela, con un $21 \%$ de sus viviendas damnificadas, Río Hurtado (17\%), Illapel (15\%), Punitaqui (9\%) y Monte Patria (8\%). Mientras que los materiales más dañados fueron adobe ( $29 \%$ de las viviendas encuestadas fueron dañadas), albañilería (25\%) y madera (11\%). Cabe destacar que la encuesta del Gobierno consta de 9317 viviendas evaluadas, de las cuales 7285 presentan algún tipo de daño. Además, el 31\% del total no tiene identificación del material de construcción y tan solo 2336 corresponden a adobe (Gobierno de Chile, 2015), dentro de un total de 12792 viviendas de este material en las comunas con estaciones sismológicas.

\section{Conclusiones}

Las razones espectrales calculadas a partir de sismos y de vibraciones ambientales son consistentes aun cuando se aprecia una degradación de rigidez producto de la magnitud del evento sísmico, mostrando el peak del terremoto un periodo más alto respecto al promedio de las curvas.

La información geológica de la Tabla 2 es consistente con las HVSR de la Figura 2, ya que gran parte de las estaciones se encuentra sobre depósitos de suelo rígidos o afloramientos rocosos. Los resultados muestran que los periodos predominantes de vibración de los suelos 
de la mayoría de las estaciones son menores a $0.4 \mathrm{~s}$, lo que concuerda con que el material más dañado durante el terremoto fuera el adobe, aun cuando su participación en la totalidad de viviendas en la región no supera el 9\%, aproximadamente.

Monte Patria parece ser un caso excepcional, puesto que la aceleración máxima registrada es más del doble que en otras estaciones y no se identifica un efecto notorio de degradación de rigidez en las curvas H/V. Esto puede implicar un efecto de amplificación topográfica que se debe estudiar con más detalle.

Las intensidades calculadas para el terremoto de Illapel 2015, a pesar de incluir pocas viviendas, son menores a las reportadas para el terremoto de Punitaqui 1997.

\section{Referencias}

Aguirre, L. y Egert, E. (1970). Geología del cuadrángulo Lambert (La Serena), Región de Coquimbo. Instituto de Investigaciones Geológicas, Chile. Carta Geológica $N^{\circ} 23$

Beck S, Barrientos S, Kausel E, Reyes, M. (1998) Source characteristics of historic earthquakes along the central Chile subduction zone. Journal of South American Earth Sciences 11(2): 115-129.

Börgel, R. (1983). Geomorfología (Geografía de Chile). Instituto Geográfico Militar. Vol. 2

Díaz, O. (2001). Estudio de los efectos de las condiciones locales en el terremoto de Punitaqui 1997. Memoria para optar al título de Ingeniero Civil, Universidad de Chile

El Mercurio (2007). Chile tiembla otra vez. Santiago, Chile, 18 de noviembre de 2007. p. D10

García, J.M. (1999). Hidrogeología del sector de Salamanca, Provincia de Choapa, IV Región. Memoria para optar al título de Geólogo, Universidad de Chile

GEER (2015). Geotechnical reconnaissance of the $2015 \mathrm{Mw}$ 8.3 Illapel, Chile Earthquake. Geotechnical Extreme Events Reconnaissance Association

Gobierno de Chile (2015). Plan de Reconstrucción Región de Coquimbo 2015. Ministerio de Vivienda y Urbanismo, http:// www.minvu.cl/opensite_20151002093225.aspx
Greve, F. (1946). Descripción de los principales efectos y ubicación del epicentro de los sismos destructores sentidos en Chile en los años 1942-43-44-45 y 46. Instituto Sismológico de la Universidad de Chile

Idini, B.R. (2016). Curvas de atenuación para terremotos intraplaca e interplaca en la zona de subducción chilena. Tesis para optar al grado de Magíster, Universidad de Chile

Konno, K. and Ohmachi, T. (1998). Ground-motion characteristics estimated from spectral ratio between horizontal and vertical components of microtremor. Bulletin of the Seismological Society of America 88(1), 228-241

Lemoine A, Campos J, Madariaga R. (2001). Evidence for earthquake interaction in the Illapel Gap of central Chile. Geophysical Research Letters 28(14): 2743-2746.

Lermo, J. and Chávez-García, F.J. (1993). Site effect evaluation using spectral ratios with only one station. Bulletin of the Seismological Society of America 83(5): 1574-1594

Medvedev, S., Sponheuer, W. und Kárnik, V. (1964). Neue seismische Skala. Deutsche Akademie der Wissenschaften zu Berlin 77

Monge, J. y Astroza, M. (1989). Metodología para determinar el grado de intensidad a partir de los daños. 5as Jornadas Chilenas de Sismología e Ingeniería Antisísmica, Santiago, vol.1, 483-492

Morales, R.M., Olano, A.S., Cabrejos, R.T. y Morales, O. (1980). Estudio sísmico de construcciones de adobe de dos pisos. Anales III Congreso Nacional de Ingeniería Civil, Perú

Mpodozis, C. y Cornejo, P. (1988). Geología de la Hoja de Pisco Elqui, Región de Coquimbo. Servicio Nacional de Geología y Minería. Carta Geológica $N^{\circ} 68$

Moscoso, R., Nasi, C. y Salinas, P. (1982). Geología de la Hoja Vallenar y parte norte de La Serena, regiones de Atacama y Coquimbo. Servicio Nacional de Geología y Minería. Carta Geológica $N^{\circ} 55$

Nakamura, Y. (1989). A method for dynamic characteristics estimation of subsurface using microtremor on the ground surface. Railway Technical Research Institute 30(1): 25-33

ONEMI (1997). Informes del terremoto de Punitaqui de 1997. Oficina Nacional de Emergencia del Ministerio del Interior y Seguridad Pública 
Pardo, M., Comte, D., Monfret, T., Boroschek, R. and Astroza, M. (2002). The October 15, 1997 Punitaqui Earthquake (Mw = 7.1): a destructive event within the subducting Nazca plate in central Chile. Tectonophysics 345(1): 199-210

Piñones, A.R. (2002). Efectos del sismo del 14 de octubre de 1997 en la cuidad de Ovalle. Memoria para optar al título de Ingeniero Civil, Universidad de Chile

Rivano, S. y Sepúlveda, P. (1991). Geología de la Hoja de Illapel, Región de Coquimbo. Servicio Nacional de Geología y Minería. Carta Geológica Nº 69

Rosales, G.A. (1988). Geología del área de Combarbalá - San Lorenzo (3100' - 31 15' Latitud Sur), Región de Coquimbo. Memoria para optar al título de Geólogo, Universidad de Chile
Ruiz, S., Klein, E., del Campo, F., Rivera, E., Poli, P., Metois, M., Vigny, C., Baez, J.C., Vargas, G., Leyton, F., Madariaga, R. and Fleiout, L. (2016). The seismic sequence of the 16 September 2015 Mw 8.3 Illapel, Chile, Earthquake. Seismological Research Letters 87(4): 789-799

Sáez, M.A. (2016). Correlación cruzada de ruido sísmico para la obtención de perfiles profundos de velocidad de onda de corte en la cuenca de Santiago. Tesis para optar al grado de Magíster en Ingeniería Sísmica, Universidad de Chile

Thomas, H. (1967). Geología de la Hoja de Ovalle, Provincia de Coquimbo. Instituto de Investigaciones Geológicas, Chile. Boletín $\mathrm{N}^{\circ} 23$ 